\title{
CIRCADIAN RHYTHMS IN THE LIMULUS VISUAL SYSTEM ${ }^{1}$
}

\author{
ROBERT B. BARLOW, JR.
}

Institute for Sensory Research, Syracuse University, Syracuse, New York 13210

Received September 14, 1982; Revised November 22, 1982; Accepted November 22, 1982

\begin{abstract}
A circadian clock in the Limulus brain generates efferent optic nerve activity at night. The endogenous activity begins near dusk, continues during the night, and stops near dawn. Approximately 10 to 20 efferent fibers in each lateral optic nerve trunk fire in close synchrony with one another and with the efferent fibers in the opposite nerve trunk producing bursts of nerve impulses at night. The synchronous bursting activity indicates extensive coupling in the brain among the efferent neurons or among the circadian pacemakers that drive them.

The efferent optic nerve activity mediates circadian rhythms in retinal responses. Electroretinograms (ERGs) from both the lateral eyes and median ocelli exhibit circadian rhythms of equal phase. Sectioning the optic nerves abolishes the rhythms. When the animal is kept in darkness, the phase of the rhythm can be shifted by illuminating the lateral eyes but not the median ocelli. The endogenous rhythm persists for at least 1 year in darkness without attenuation and without substantial changes in the circadian period. Across a large population of animals the duration of the circadian period ranges from 22.2 to $25.5 \mathrm{hr}$, with a mean value of $23.9 \pm 0.7 \mathrm{hr}(n=75)$.

The nighttime increase in ERG amplitude represents a 20- to 100-fold increase in retinal sensitivity. Most of the increased sensitivity results from an increase in the number of photons absorbed by the photoreceptors at night. A small component may be due to an increase in gain. Pulses of current delivered to the lateral optic nerve during the day mimic the effects of endogenous efferent activity and transform the eye to the nighttime state.

The Limulus visual system has evolved numerous mechanisms for adapting visual sensitivity to daily fluctuations in the photic environment. A key to understanding the organization of the visual system is the circadian clock and its associated neural circuitry.
\end{abstract}

Sensory organs encode and transmit information to the brain about the physical environment. In many animals the brain in turn transmits signals back to the sensory organs. This central feedback may tune sensory receptors to specific stimuli, adapt sensory function to changes in the physical environment, or control metabolic processes in sensory organs. Efferent neural pathways or circulating neurohormones may mediate the feedback from the brain.

The Limulus visual system provides a clear example of the brain's modulation of a sensory input. Efferent optic nerve fibers transmit neural signals from the brain to the lateral eyes at night (Barlow et al., 1977). The efferent signals change the structure of retinal cells (Bar-

\footnotetext{
${ }^{1}$ This research was supported by National Institutes of Health Grant EY-00667 and National Science Foundation Grant BNS 82-41893. I thank Stanley J. Bolanowski, Jr., Michael L. Brachman, and Lionel A. Galway, Jr. for assistance during early stages of the work. I also thank Robert and Joseph Fladd for technical assistance and E. Zahora for typing the manuscript. I wish to acknowledge Ranjan Batra, Steven C. Chamberlain, and Leonard Kass for helpful discussions.
}

low and Chamberlain, 1980; Chamberlain and Barlow, 1977), increase the field of view of ommatidia (Barlow et al., 1980a), control photoreceptor metabolism (Chamberlain and Barlow, 1979), decrease photoreceptor noise (Kaplan and Barlow, 1980), and decrease retinal inhibitory interactions (Batra and Barlow, 1982). All effects combine to increase visual sensitivity.

A circadian clock in the brain generates the efferent optic nerve activity. When the animal is maintained in constant darkness, the clock initiates efferent activity at dusk, maintains the activity throughout the night, and stops generating activity near dawn. All major photoreceptor organs-lateral eyes, medial ocelli, and ventral photoreceptors-receive efferent activity from the clock at night (Eisele et al., 1982). In addition, the circadian clock enables one photoreceptor organ to modulate the sensitivity of another at night but not during the day (Barlow et al., 1980a). It is apparent that Limulus has evolved elegant mechanisms for adapting visual sensitivity to fluctuations in ambient illumination caused by the earth's rotation. The circadian rhythms in visual sensitivily may play a role in the animal's mating behavior (Barlow et al., 1982). 
I present here some of the properties of the circadian rhythms in visual sensitivity as measured by the electroretinogram (ERG). I describe the characteristics of rhythms that are free running in darkness and the effect of light pulses on the phase of the rhythms. I also describe the characteristics of the efferent optic nerve activity that links the circadian pacemaker in the brain with the photoreceptors in the eye. A preliminary report of portions of this work has appeared elsewhere (Barlow et al., 1977).

\section{Materials and Methods}

The experiments were performed at two locations. Over the past 6 years numerous experiments were carried out during the months of May to August at the Marine Biological Laboratory, Woods Hole, Massachusetts. Horseshoe crabs were collected in the environs of Woods Hole and stored in tanks that were continuously flushed with seawater from Woods Hole harbor. The tanks were located outdoors so that the animals received natural diurnal changes of sunlight. The experimental animals ranged from juveniles ( $1.5 \mathrm{~cm}$ across the carapace) less than 1 year old to adult males $(20$ to $25 \mathrm{~cm}$ across the carapace) more than 6 years old. Experiments were also performed in Syracuse with animals shipped from the Marine Biological Laboratory, Woods Hole, the Gulf Specimen Company, Panacea, Florida, and from Shark River, New Jersey. In Syracuse the animals were kept in artificial seawater (ASW) (Instant Ocean, Aquarium Systems, Inc., Eastlake, OH), fed regularly, and exposed to the natural diurnal changes of sunlight.

Recording the electroretinogram. An animal which had been maintained in a natural light-dark cycle was clamped to a rigid platform in an aquarium located in a lightproof, shielded cage. The aquarium was filled with ASW to the level of the lateral eyes, and the gill structure was continuously washed with aerated ASW. In the Syracuse experiments the temperature $\left(18\right.$ to $\left.20^{\circ} \mathrm{C}\right)$ and $\mathrm{pH}$ ( 7 to 7.5 ) of the aquaria were approximately the same as those in the holding tanks. In the Woods Hole experiments the aquaria contained filtered seawater from the outdoor tanks in which the animals were stored.

ERGs were recorded with a corneal electrode fabricated from a Pasteur capillary pipette. The tip of the pipette was constricted under a flame and then filled with a bundle of glass fibers or nylon threads. A platinum wire was inserted in the open end, and the pipette was filled with ASW and sealed with a rubber bulb. The tuft of glass fibers or nylon threads at the electrode tip was brought into contact with the cornea, and the platinum wire was attached to a low noise, differential amplifier (gain $10^{4}$, bandpass 3 to $300 \mathrm{~Hz}$, Electronics Laboratory, Rockcfcller University). Capillary action at the tip maintained electrical contact with the cornea sufficiently well to yield stable ERG recordings from a single eye for at least a 2-month period.

ERGs are used exclusively in this study as measures of retinal response. In some invertebrates the ERG contains components generated by the neural activity of both receptor and postreceptor cells. This does not seem to be the case for the Limulus eye. Hartline (1935) showed that the extracellular potential recorded from a single isolated ommatidia was similar to that recorded from the surface of the cornea. L. Kass (personal communication) found that blocking the generation of optic nerve activity by perfusing the eye with tetradotoxin did not change the waveform of the ERG. Thus it seems reasonable to conclude that the components of the ERG reflect only the activity of photoreceptor cells. In this study the peakto-peak amplitude of the ERG is used as a relative measure of photoreceptor response.

Optical stimulation. One method for delivering brief flashes to the lateral eyes employed a white Teflon screen placed between the eye and a light source. Diffuse retinal illumination was accomplished by placing the screen 2 $\mathrm{cm}$ in front of the eye with the plane of the screen perpendicular to the optic axis of the center portion of the eye as judged by the pseudopupil. Light from a tungsten filament source was projected on the screen by a large fiberoptic bundle (diameter $2.5 \mathrm{~cm}$ ). This technique provided uniform illumination of all but the most peripherally located ommatidia in the eye (Barlow and Fraioli, 1978). It was used primarily in the experiments in Figures 11 and 12 that investigated the sensitivity of the retina by measuring the intensity-response function of the ERG response. In both figures the maximum intensity incident on a single ommatidium $(\log I=0)$ was estimated to be about $3 \times 10^{11}$ photons/sec between 400 and $700 \mathrm{~nm}$ measured with a calibrated photodiode, PIN10D (United Detector Technology, Inc., Santa Monica, CA).

A second optical stimulator utilized green light-emitting diodes (LEDs) (Model XC 5549-G24, Xciton Corp., Latham, NY). The output intensity of the LEDs increases linearly with current over a 3-log-unit range and remains stable over extended periods of time. Thus they prove to be a convenient and relatively inexpensive means for long term measurements of the circadian rhythms in the Limulus visual system.

Recording and data analysis. Many of the experiments reported here were carried out under the control of a microprocessor (AIM-65, Rockwell) together with an A/D converter and a current driver to control the LEDs (ISR Electronics Shop, Syracuse University). The AIM65 was programmed to keep the time of day, deliver current pulses to the LEDs at specified intervals, and analyze the ERG waveform. After each test flash the microprocessor printed the date, time of day, peak-topeak amplitude of the ERG, and latency to the first peak of the ERG. When necessary, the microprocessor triggered the shutter of the tungsten optical system and/or gated a chart recorder (Model 22D, Gould Brush). The system was well suited for long term experiments.

Figure 1 shows how the circadian rhythms of the ERG responses were analyzed. Plotted are the peak-to-peak amplitudes of the ERG from a lateral cye as a function of the time of day beginning on the day the animal was placed in darkness in the shielded, lightproof recording cage. Note that the daytime amplitude of the ERG was higher on days 2 and 3 than on day 1 . This point will be discussed later. After the first day in darkness, the circadian changes in ERG amplitude generally stabilize and remain stable for periods of up to 2 months.

Figure 1 shows that the animal's "subjective night length" is defined as the number of hours the ERG amplitude exceeds $25 \%$ of the difference between the 


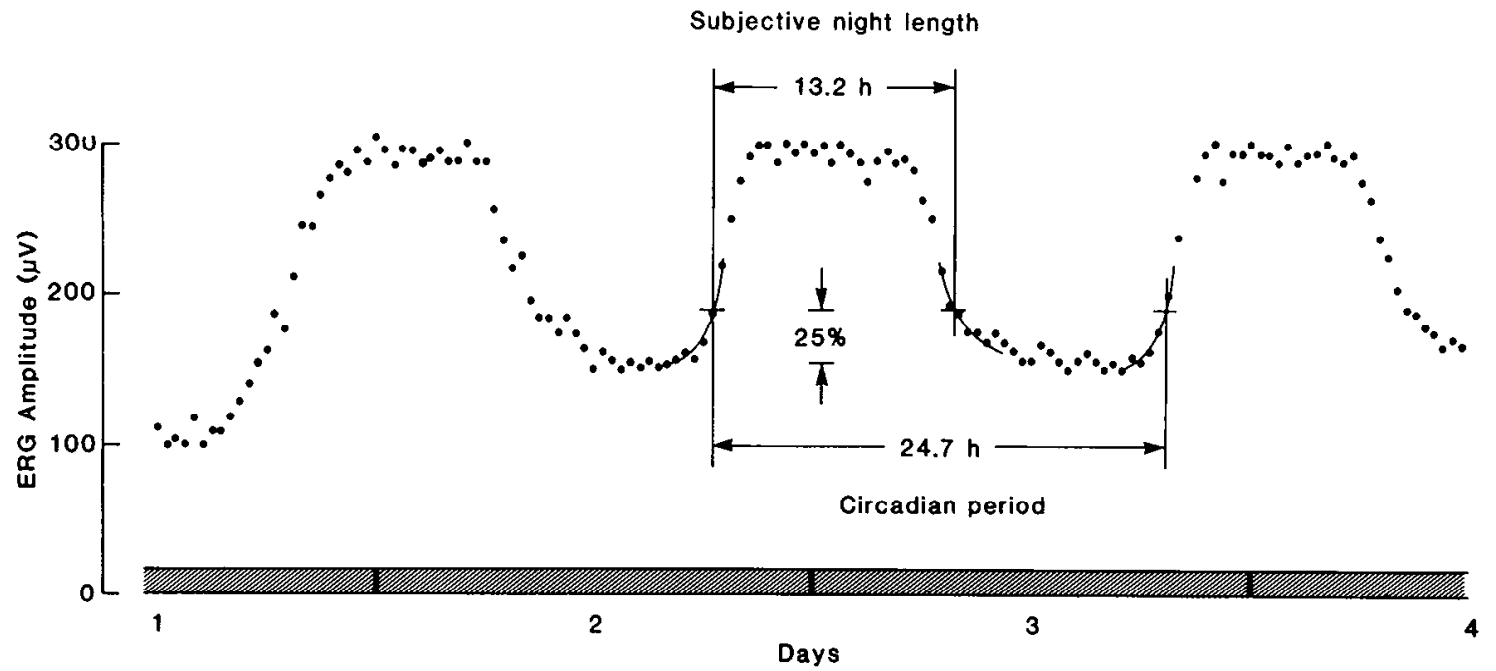

Figure 1. Method of analyzing circadian rhythms of the electroretinogram. Points give the peak-to-peak amplitude of the ERG elicited by $100-\mathrm{msec}$ flashes of light presented every $30 \mathrm{~min}$. The animal was placed in darkness in the recording apparatus during the morning of day 1 . At noon the testing sequence was initiated and was continued until noon of day 4 . The animal remained in darkness throughout the 72 -hr period. Cross-hatching on the ordinate indicates darkness; vertical bars indicate midnight. As noted in the text the "subjective night length" and "circadian period" are measured after the first night in darkness. All measurements are made for a $25 \%$ increment in the amplitude of the ERG.

daytime and nighttime levels. The $25 \%$ points were determined after smooth curves were fit by eye. The choice of $25 \%$ is based on experiments which recorded simultaneously the ERG responses of one lateral and the ERG and efferent input to the other (see Fig. 9). The records indicate that the duration of efferent activity from onset at dusk to offset at dawn corresponds to the duration of elevated ERG responses when measured at the $25 \%$ level. Thus the "subjective night length" provides a useful measure of the duty cycle of the circadian pacemaker. The "circadian period" was measured as the number of hours between the intercepts at the $25 \%$ levels on sequential days.

Recording efferent optic nerve activity. The lateral optic nerve was exposed by cutting a circular hole (1.9 $\mathrm{cm}$ in diameter) in the carapace about $3 \mathrm{~cm}$ anterior to one of the lateral eyes. The optic nerve trunk runs directly under the ophthalmic ridge at this point. After clearing the nerve of surrounding tissue, it was cut, and the cut end coming from the brain was drawn into a small recording chamber which fit snuggly in the hole. The nerve was then dissected with fine needles into small strands of fibers which were then tested for efferent activity by drawing them into the glass tip $(100-\mu \mathrm{m}$ opening) of a suction electrode. The electrode and recording chamber were filled either with organ culture (Bayer and Barlow, 1978) or with ASW containing 100 units/ml of penicillin and $100 \mu \mathrm{g} / \mathrm{ml}$ of streptomycin (Kaplan and Barlow, 1975). This procedure is usually carried out near dusk, which is the time the circadian clock begins to generate efferent activity.

Optic nerve stimulation. Efferent optic nerve activity was simulated by delivering current shocks to the proximal end of the cut optic nerve. The procedure was similar to that for recording efferent activity with the exception that the proximal end of the cut nerve was drawn into the suction electrode (1-mm opening), and the lead to the suction electrode was plugged into a stimulus isola- tion unit of a pulse stimulator (Models PS1U6 and S44, Grass Instrument Co.). Pulses $1 \mathrm{msec}$ in duration were delivered to the cut nerve at the rate of 2 per sec for 30 sec every minute. This rate matches well that of the endogenous efferent activity (Barlow et al., 1977).

\section{Results}

Circadian rhythms in lateral eye response. Figure $2 a$ gives ERGs recorded from a Limulus lateral eye in situ. Brief test flashes of constant intensity were delivered to the eye every $30 \mathrm{~min}$ for a period of 3 days while the animal remained in the dark. The animal had been maintained in a natural light-dark cycle before being placed in the ERG recording apparatus. Note that on day 1 the amplitude of the ERG began to increase at 3:30 P.M., reached a maximal level about 6 P.M., remained elevated until 2:30 A.M., and then began to decrease to a low daytime level. The cycle continued on days 2 and 3 , but the time the amplitude began to increase occurred progressively later in the afternoon. The rhythmic changes had a period of $24.8 \mathrm{hr}$ as measured by the criteria in Figure 1. After 2 weeks in darkness (data not shown), the rhythm had drifted completely out of phase with the solar day.

Figure $2 b$ gives ERGs recorded from an animal that had been maintained in the dark for 1 year. Clearly an endogenous rhythm persisted after such a prolonged period of darkness. The rhythm had a period of $23.4 \mathrm{hr}$. Every week the animal was fed and the aquarium water and filters were checked under brief illumination of a darkroom safelight. Twice during the year the animal was taken from the aquarium and placed in the ERG recording apparatus. The results of each test are similar to those in Fig. 2a. Apparently the endogenous rhythm in the lateral eye ERG persists over long periods in the dark without noticeable attenuation.

Figure 3 shows the ERG data recorded continuously from the lateral eye of one animal for 50 days. Black 
a

Day 1

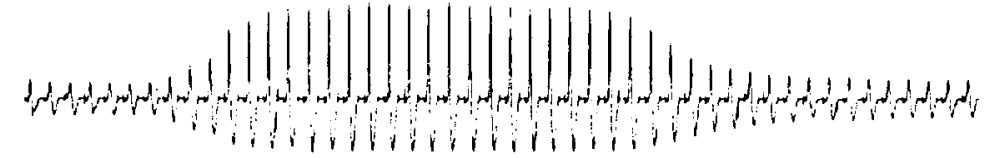

Day 2
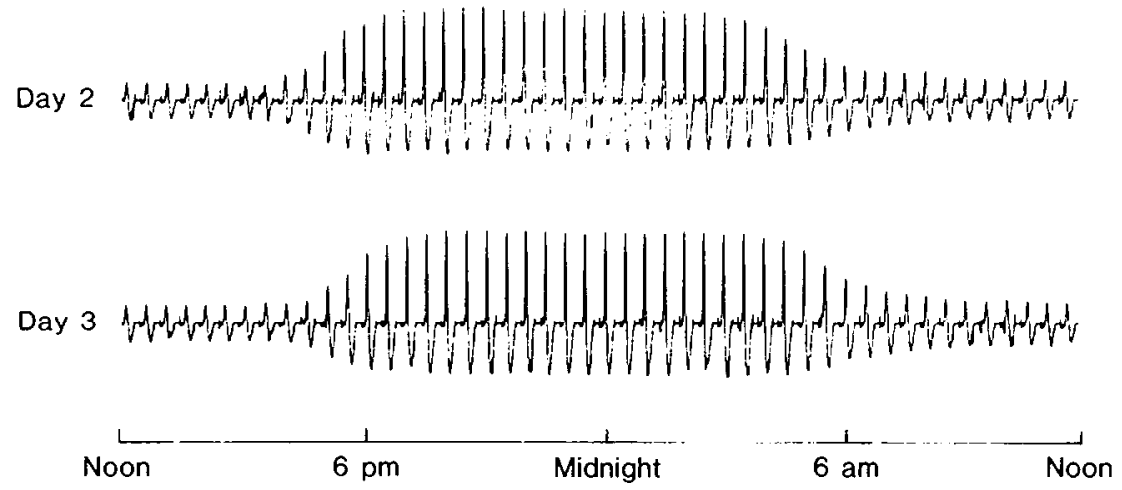

b

Day 365

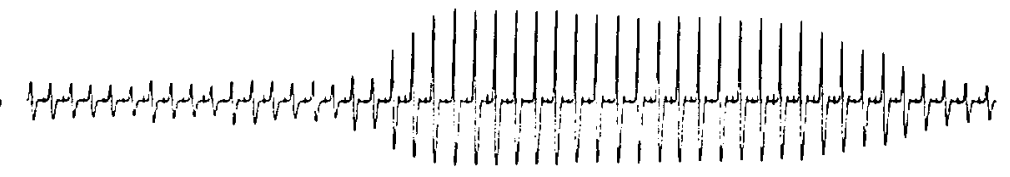

Day 366
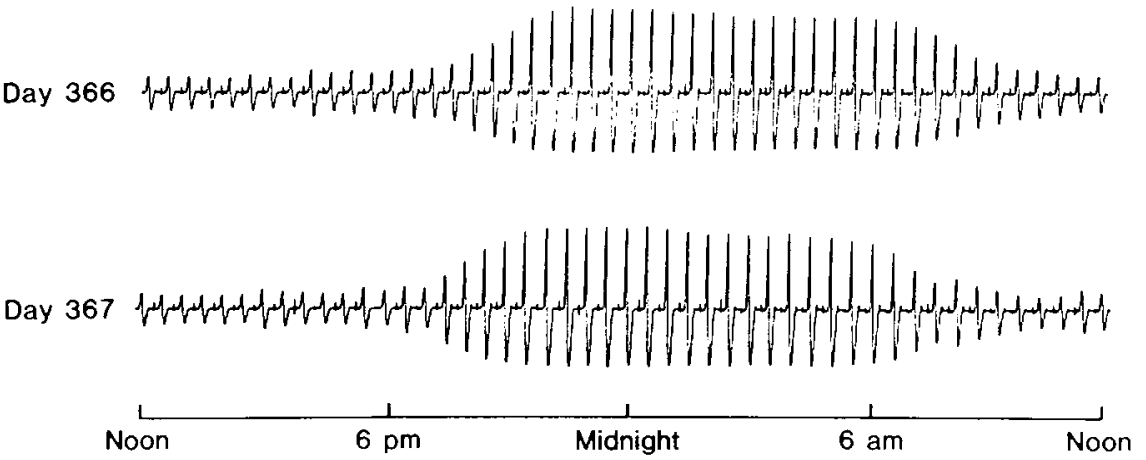

Figure 2. Circadian rhythms in the amplitude of the ERG of the lateral eye. $a, A$ continuous chart record of ERG response recorded over a 3-day period from a lateral eye of an animal placed in darkness on day 0 . At noon of day 1 the chart recorder was gated on for $700 \mathrm{msec}$. After a delay of $100 \mathrm{msec}$, a brief flash $100 \mathrm{msec}$ in duration was presented to the eye. The chart recorder stopped $500 \mathrm{msec}$ after offset of the flash. The sequence was repeated every 30 min for 3 days, with the test flash intensity held constant. The continuous record was cut into three sections in order to compare the circadian changes in ERG amplitude. The endogenous rhythm had a pexiod of $24.8 \mathrm{hr}$. The peak-to-peak ERG amplitude during the first night was $120 \mu \mathrm{V}$. The light intensity incident on a single ommatidium from the tungsten filament source was approximately $1 \times 10^{6}$ photons/flash. Data were recorded from an adult animal $(22 \mathrm{~cm}$ across the carapace). Juveniles as small as $1.5 \mathrm{~cm}$ across the carapace yielded similar results. $b$, A continuous chart record of ERG responses from a lateral eye of an animal maintained in darkness for 1 year. The recording procedure and test flash intensity were the same as in $a$, but the animal was not. The circadian rhythm of this animal had a period of $23.4 \mathrm{hr}$. Note that on day 365 the animal's subjective night begins about 9 P.M. and ends about 10 A.M. On the following days the subjective night begins progressively earlier. The peak-to-peak ERG amplitude was $130 \mu \mathrm{V}$ during the night of day 365 .

bars indicate the animal's subjective night, defined in Figure 1 as the time of day during which the ERG responses exceed a $25 \%$ increase in amplitude over the daytime level. To demonstrate clearly the periodicity of the endogenous rhythm, the ERG data for each day are plotted twice. During the first day in darkness the cyclic changes in visual response matched closely the natural light-dark cycle in which the animal had been maintained. On the following days the rhythm persisted, with a period of $23.5 \mathrm{hr}$, and within a month it had drifted completely out of phase with the solar day. After 30 days in the dark, the length of the period decreased to about $23.1 \mathrm{hr}$, producing a higher rate of drift. The visual rhythm completed a $360^{\circ}$ phase shift wihin 1.5 months. The gradual decrease in the length of the period of $0.4 \mathrm{hr}$ is the maximum change observed thus far for recordings extending up to 2 months. Longer recording sessions have not been possible. Apparently the animals cannot tolerate more than about 2 months in the recording apparatus. 
In sum, the data in Figures 2 and 3 show that the Limulus lateral eye exhibits an endogenous rhythm in ERG response with a period of about $24 \mathrm{hr}$. The rhythm persists for at least a year under conditions of constant darkness. The period of the rhythm may vary from animal to animal, but for a given animal it remains relatively constant when the animal is kept in darkness. Measurements of ERG records from 75 animals yield periods ranging in length from 22.2 to $25.5 \mathrm{hr}$, with a mean value of $23.9 \pm 0.7 \mathrm{hr}$.

Figure 4 shows that the phase of the ERG rhythm can be shifted by exposing the animal to brief periods of illumination. At midnight on December 23 the light-tight recording cage was opened to room illumination for $2 \mathrm{hr}$. On the following day the increase in ERG amplitude was delayed by $4 \mathrm{hr}$ to 10:30 P.M. For the next 8 days (December 24 to 31 ), the ERG rhythm continued in the dark with the same period that preceded the light exposure on December 23. After 1 P.M. on New Year's Eve a 2-hr exposure was repeated, and at this point in the cycle it caused a phase advance of $1 \mathrm{hr}$. A 2-hr phase advance occurred on January 5 in response to $2 \mathrm{hr}$ of illumination on the preceding day. It is apparent that the amplitude and sign of the phase shift depended on the time of exposure during the cycle.

Figure 5 plots the amplitude and sign of phase shifts as a function of the time of onset of a brief period of illumination during the animal's circadian cycle. The open circles were taken from the experiment in Figure 4. The solid circles and triangles were taken from experiments that were similar to those in Figure 4 with the exception that the light pulses to shift the phase were separated by 2 days, whereas 3 days was the shortest interval in the experiment in Figure 4. Phase shifts, both advances and delays, seem to be complete on the day following the brief period of illumination (Fig. 4). No evidence was obtained for the transient effects observed in circadian rhythms of other animals (Pittendrigh and Bruce, 1959).

The squares in Figure 5 come from a similar experiment except that, after four tests to shift the phase, the median ocelli were excised and to additional tests were carried out (squares with diagonal slashes). In this experiment excising the ocelli did not reduce the effectiveness of light pulses to shift the phase of the rhythm. In other experiments ocellar illumination alone proved relatively ineffective. Although these few experiments are not conclusive, they suggest that the activity of the lateral eyes or photosensitive organs other than the ocelli synchronize the endogenous rhythm to the natural lightdark cycle.

Figure 5 shows that light pulses presented during the

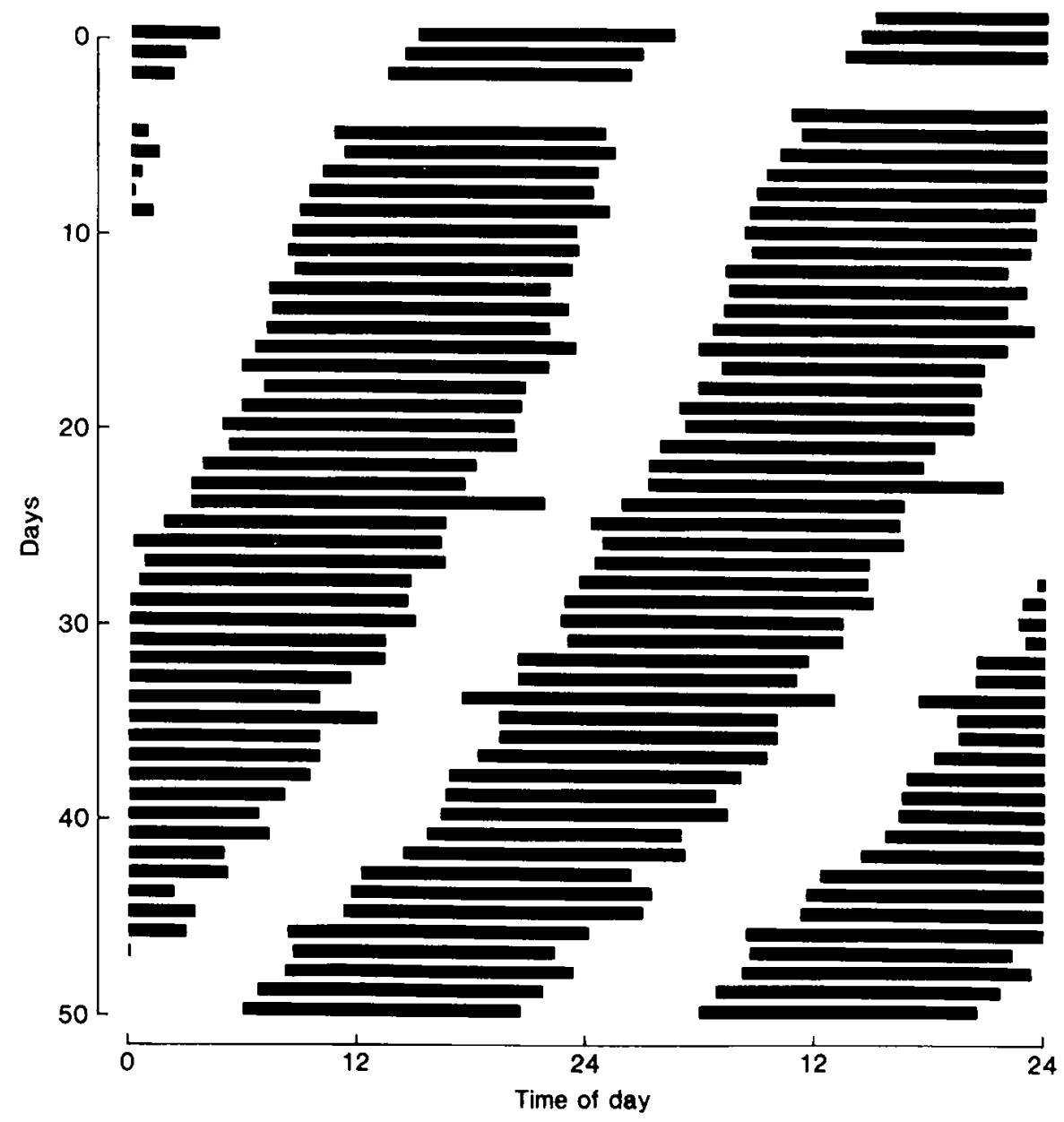

Figure 3. Circadian rhythm in lateral eye ERG recorded for 50 days in constant darkness. Black bars indicate the periods of elevated ERG amplitude defined as subjective night lengths in Figure 1. To enhance the periodicity of the rhythm, the data were plotted twice on the abscissa, yielding a double folded configuration. The period of the circadian rhythm was $23.5 \mathrm{hr}$ during the first 4 weeks in darkness, and then it decreased to about $23.1 \mathrm{hr}$ for the remaining 3 weeks. Two days of recordings were lost at the beginning of the experiment. 


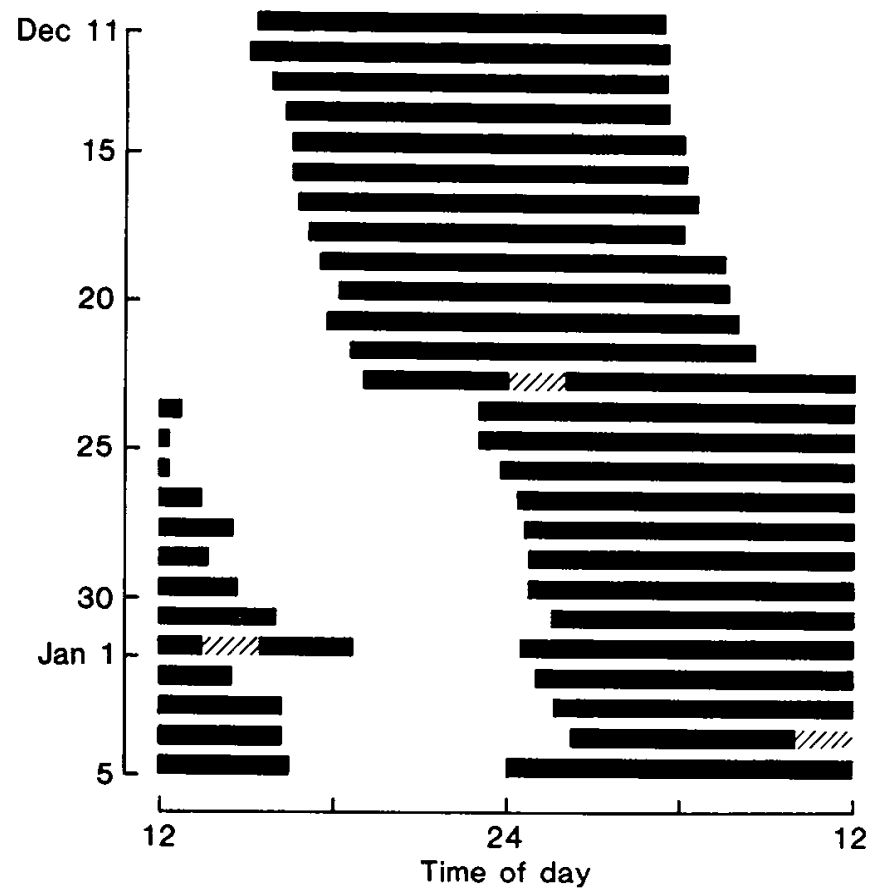

Figure 4. Phase shifts of the circadian rhythm in lateral eye ERG. Experimental conditions are the same as in Figure 3, with the exception that on three occasions the dark recording cage was opened to expose the animal to 2 -hr periods of room illumination. Each exposure shifted the phase. The exposure presented during the middle of the animal's subjective night on December 23 delayed the phase of the rhythm. The two following exposures advanced the phase.

animal's subjective night cause maximum phase shifts. Pulses presented before midnight (circadian time 12 to $18 \mathrm{hr}$ ) delayed the phase of the cycle, and pulses after midnight (circadian time 18 to 24 ) advanced the phase. One pulse at midnight had no effect. Phase shifts were relatively small for pulses presented during the subjective day. These results are similar to the phase-response characteristics of cyclic phenomena from a variety of organisms and are generally regarded as evidence for a circadian clock.

In sum, visual responses of the Limulus lateral eye exhibit an endogenous rhythm when the animal is maintained in constant darkness. The period has a rhythm of about $24 \mathrm{hr}$, and its phase can be shifted by pulses of light. Because such results characterize numerous circadian phenomena, it seems reasonable to conclude that an endogenous circadian clock modulates visual function in Limulus.

Efferent optic nerve fibers mediate the circadian rhythms. Figure 6 shows that the lateral eyes and median ocelli exhibit synchronous circadian rhythms and that cutting the optic nerve abolishes the rhythms. The diagram on the left shows the experimental arrangment for recording both lateral eyes and the right median ocellus. Space limitations impaired placement of another electrode on the left ocellus. The animal was mounted on a platform in an aquarium as described under "Materials and Methods." But before placing the aquarium in the dark, three holes were drilled through the carapace exposing the lateral and median optic nerve trunks. Snares of surgical thread were inserted around each nerve trunk, then gauze pads were placed over the holes and tucked under the carapace to prevent bleeding and to allow access to both ends of each snare. Light pipes and corneal electrodes were aligned and the recording cage was closed, leaving the animal in complete darkness. Every $30 \mathrm{~min}$, brief flashes of light were delivered to the eyes and the three ERG responses were recorded. The solid circles on the right plot the peak-to-peak amplitude of the ERG in microvolts as a function of time in the dark in days.

After the animal was placed in the dark, the ERGs from the three eyes exhibited synchronous circadian rhythms. At midnight on day 3 , the recording cage was opened and the snare pulled to sever the left optic nerve trunk. The operation was performed without exposing the animal to light and without stretching the nerve. After the optic nerve was cut, the ERG amplitude began to decrease following an exponential time course with a time constant of $0.78 \mathrm{hr}$. Within $2 \mathrm{hr}$ the amplitude reached the low, dark-adapted daytime level. No circadian changes were detected after the nerve was cut. At midnight on days 5 and 7 the operation was repeated for the median and right-lateral optic nerve trunks. The results were the same: cutting a nerve trunk at night reduced the ERG amplitude to the daytime level and

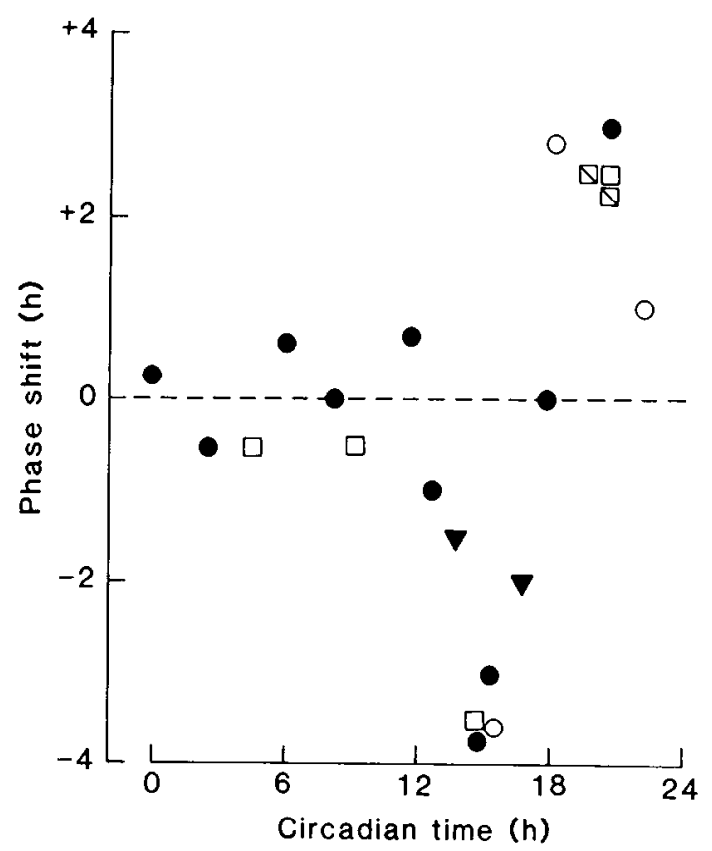

Figure 5. Phase-response curve for the circadian rhythm of the lateral eye ERG. On the ordinate are plotted the magnitude and sign of phase shifts evoked by light exposures of $2 \mathrm{hr}$ in duration. Phase advances are indicated as positive and phase delays as negative. On the abscissa are plotted the times of onset of the 2-hr exposure. The circadian time of $0 \mathrm{hr}$ (CT 0 ) is defined as the animal's subjective dawn according to the criteria in Figure 1. For example, on day 3 in Figure 1 CT 0 occurred at 7:53 A.M. The data summarize the results of four experiments. The open circles were taken from the experiment in Figure 4. The solid circles and triangles were taken from similar experiments, with the exception that the light pulses to shift the phase were separated by only 2 days. The squares came from an experiment in which the median ocelli were excised (squares with diagonal slashes) after four tests were carried out under normal conditions. 
abolished subsequent circadian changes in response. In other experiments, cutting the optic norve at noon caused no immediate change in ERG amplitude but abolished any subsequent changes. These results indicate that the increased amplitude of visual responses at night is caused by a circadian clock which generates efferent activity in the median and lateral optic nerves.

Efferent optic nerve activity. Efferent activity can indeed be recorded at night from the lateral optic nerves. Figure 7 shows trains of efferent activity recorded simultaneously from fibers in the proximal stumps of the cut lateral optic nerve trunks. In this experiment only a single train of efferent impulses could be recorded from the right optic nerve, whereas regular bursts of impulses were recorded from the left optic nerve. The nature of the recording is determined by the dissection. The efferent fibers are small, fragile, and difficult to locate. However, when found, the efferent activity is generally characterized by repetitive bursts of impulses.

The efferent fibers in both optic nerves fired in near synchrony. The single efferent fiber in the right optic nerve fired an impulse each time a burst of efferent impulses was fired in the left optic nerve. The single fiber discharge was synchronous with the bursts, but not with any specific spikes within the bursts. Nine spikes can be identified in each burst from the left optic nerve. Their order of firing was not fixed. Other experiments yielded up to 12 spikes in a burst. The data indicate that a lateral optic nerve contains a small number of efferent fibers (10 to 20) which fire in near synchrony with one another and with a separate group of efferent fibers in the opposite lateral optic nerve.

Figure 8 gives traces of efferent activity recorded over a 24-hr period from a lateral optic nerve trunk. The efferent activity was maximal in the early evening hours when the frequency of bursts reached about 2 per sec. Lower burst rates occurred during the late evening and early morning hours. No activity was recorded during the day ( 7 A.M. to 5 P.M.). Note that before midnight the duration of individual bursts was shorter than after midnight. For example, it is difficult to determine whether the efferent spikes in the 6 A.M. trace constitute

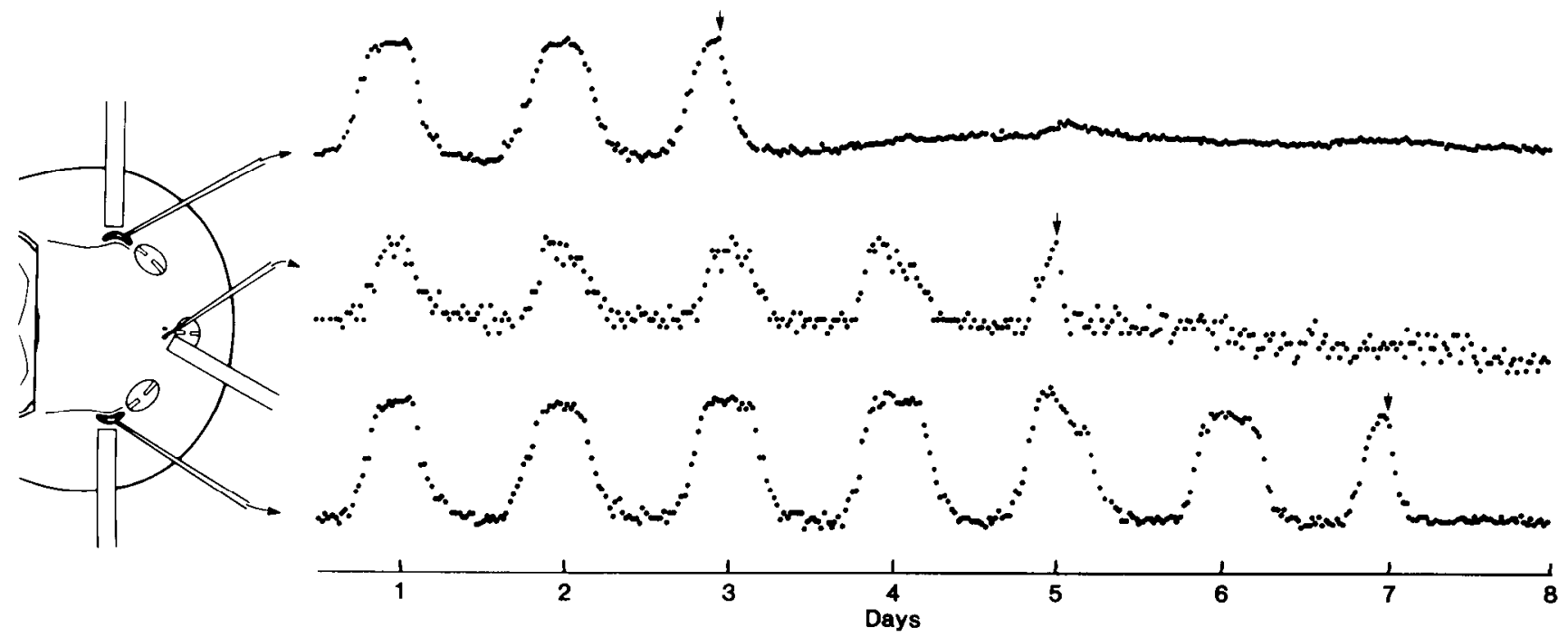

Figure 6. Lateral eyes and median ocelli exhibit synchronous circadian rhythms. On the left is a diagram of the experimental preparation showing the placement of the corneal electrodes for recording the ERGs from both lateral eyes and the right median ocellus. Also indicated are the placement of the four optic light pipes for delivery test flashes and the location of holes in the carapace for sectioning the optic nerve trunks. On the right are plotted the peak-to-peak amplitude of the ERG as a function of time of day for 8 days while the animal remained in the dark. For both lateral eyes the ERG amplitudes increased from about 40 $\mu \mathrm{V}$ during the day to $200 \mu \mathrm{V}$ at night. The median ocellar response increased from about $35 \mu \mathrm{V}$ to $120 \mu \mathrm{V}$. Sectioning the optic nerve trunks abolished the circadian rhythms. The operations were carried out in the dark by pulling snares that had been implanted around the nerve trunks before the animal was placed in the dark.

Right optic nerve

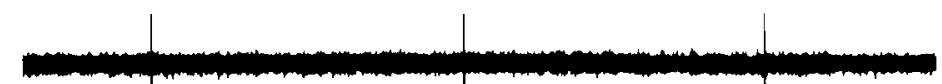

Left optic nerve

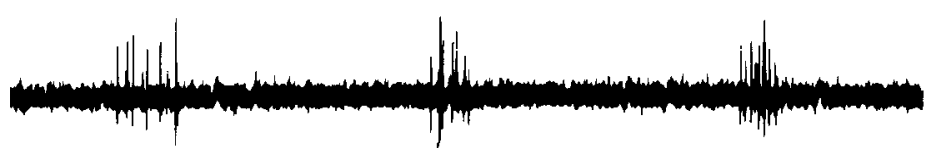

Figure 7. Efferent optic nerve activity. Traces give simultaneous recordings of efferent activity from the right and left lateral optic nerves in situ taken at 8 P.M. with the animal in darkness. Bursts of efferent impulses were recorded from the proximal stump of the left optic nerve. Burst rate is about $2 / \mathrm{sec}$. Nine spikes can be identified in each burst. Up to 12 have been detected in other experiments. Only a single train of impulses could be recorded from the right optic nerve. Recordings are 3.5 sec in duration. 
Noon

$$
3 \mathrm{pm}
$$

$6 \mathrm{pm}$

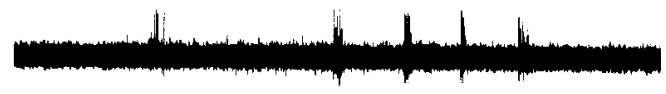

$9 \mathrm{pm}$

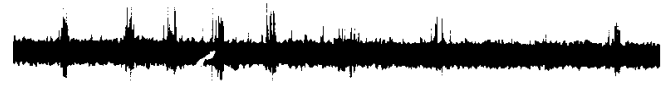

Midnight

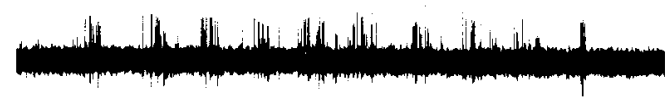

3 am

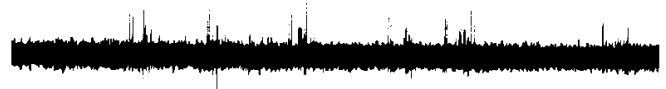

6 am

9 am

\section{Noon}

Figure 8. Efferent optic nerve activity recorded in situ over a 24 -hr period. The animal remained in the dark throughout the recording period. Note that distinct bursts of efferent impulses occurred in the early evening hours. After midnight the bursts are less distinct and the overall activity is lower. Each trace is $8 \mathrm{sec}$ in duration.

one or more bursts; however, it is clear that the spikes in the 6 P.M. trace were fired in five distinct bursts. The bursts that characterize efferent activity indicate that the efferent neurons on one side of the brain are coupled with one another and also are coupled with efferent neurons on the opposite side of the brain. The neural coupling among efferent neurons seems to change during the circadian cycle.

The characteristics of efferent optic nerve fibers can be summarized as follows:

1. Each lateral optic nerve contains a small but separate group of efferent fibers (approximately 10 to 20).

2. Efferent fibers fire in bursts at rates up to 2 bursts/ sec.

3. Each efferent fiber fires one spike in a burst.

4. Bursts occur synchronously in the lateral optic nerves.

5. Coupling among efferent neurons seems to decrease during the circadian cycle.

6. Efferent activity undergoes a circadian rhythm.

The circadian rhythm of efferent optic nerve activity coincides with the circadian rhythm of the amplitude of the ERG. Figure 9 gives the level of efferent activity recorded from several fibers in the right optic nerve and the amplitude of the ERG recorded from the left lateral eye. The animal remained in constant darkness throughout the experiment except for brief flashes delivered every 15 min to elicit the ERG. During the first night, both the efferent activity and ERG amplitude remained elevated until 5:30 A.M., at which time the efferent activity decreased rapidly toward zero and the ERG decayed slowly to the dark-adapted daytime state. No efferent activity was detected for the next $11 \mathrm{hr}$, and the ERG remained in the low daytime state. At 5:00 P.M. endogenous efferent activity appeared and the ERG amplitude began to rise. Both reached high nighttime levels by 7:30 P.M. and did not begin to decline until 5:15 A.M. Efferent activity stopped by 6:15 A.M., and the ERG amplitude again declined slowly to the daytime state. Efferent activity could not be detected during the day until 3:15 P.M., when a 2-min period of activity was recorded. Note that the ERG amplitude increased slightly after the burst of efferent activity (3:30 to 4:00 P.M.) and then decayed back to the daytime state by 4:15 P.M. Sustained efferent activity began at 4:45 P.M., at which time the ERG amplitude began to increase again. The strong correlation between endogenous efferent activity and the increase in ERG amplitude is further evidence that efferent activity mediates the circadian changes in lateral eye response.

Optic nerve stimulation. Figure $10 a$ shows that pulses of current (dark bars) delivered during the day to the optic nerve in situ increased the amplitude of the ERG. The current pulses were delivered to the distal end of the cut optic nerve at the rate of $2 \mathrm{sec}^{-1}$ for $30 \mathrm{sec}$ every min. This rate approximates that of the bursts of efferent nerve impulses recorded during the early evening hours (see Fig. 7). After $1 \mathrm{hr}$ of nerve shock the ERG amplitude had increased to $350 \mu \mathrm{V}$, which is 16 times the darkadapted daytime value. The amplitude remained high during the next $2 \mathrm{hr}$ of nerve shock and, when the pulses were turned off, decayed slowly, reaching a low steady value within $3 \mathrm{hr}$. The experiment was interrupted for 30 min during the period of elevated sensitivity (crosshatching) to measure the intensity-response function of the ERG. The measurement was repeated $3 \mathrm{hr}$ and 50 min after shock offset. The intensity-response functions are presented in Figure 12 and will be discussed below.

Figure $10 b$ shows the results of a second episode of optic nerve shock that started $5 \mathrm{hr}$ after shock offset in Figure $10 a$. Note that the time scale is compressed in $b$. In the second episode the ERG amplitude again increased and remained elevated throughout the 12-hr period of shock. However, toward the end of the period a slight decrement occurred. After shock offset, the ERG again decayed to a low, steady value. Current pulses delivered to the nerve for a third time (Fig. 10c) again increased ERG amplitude but not to the previous levels. After $2 \mathrm{hr}$ of shock the amplitude began decreasing, and about $4 \mathrm{hr}$ before the shock was turned off the ERG amplitude had reached a low value close to that recorded before shock onset. At the termination of the experiment an intensityresponse function was again measured (cross-hatching at $48 \mathrm{hr}$ ).

Optic nerve shock can also increase the ERG amplitude recorded from an excised lateral eye. Figure 10d shows the results of an experiment using the same illumination, recording, and nerve shock conditions as in $a$ to $c$, but with a lateral eye that had been excised under 


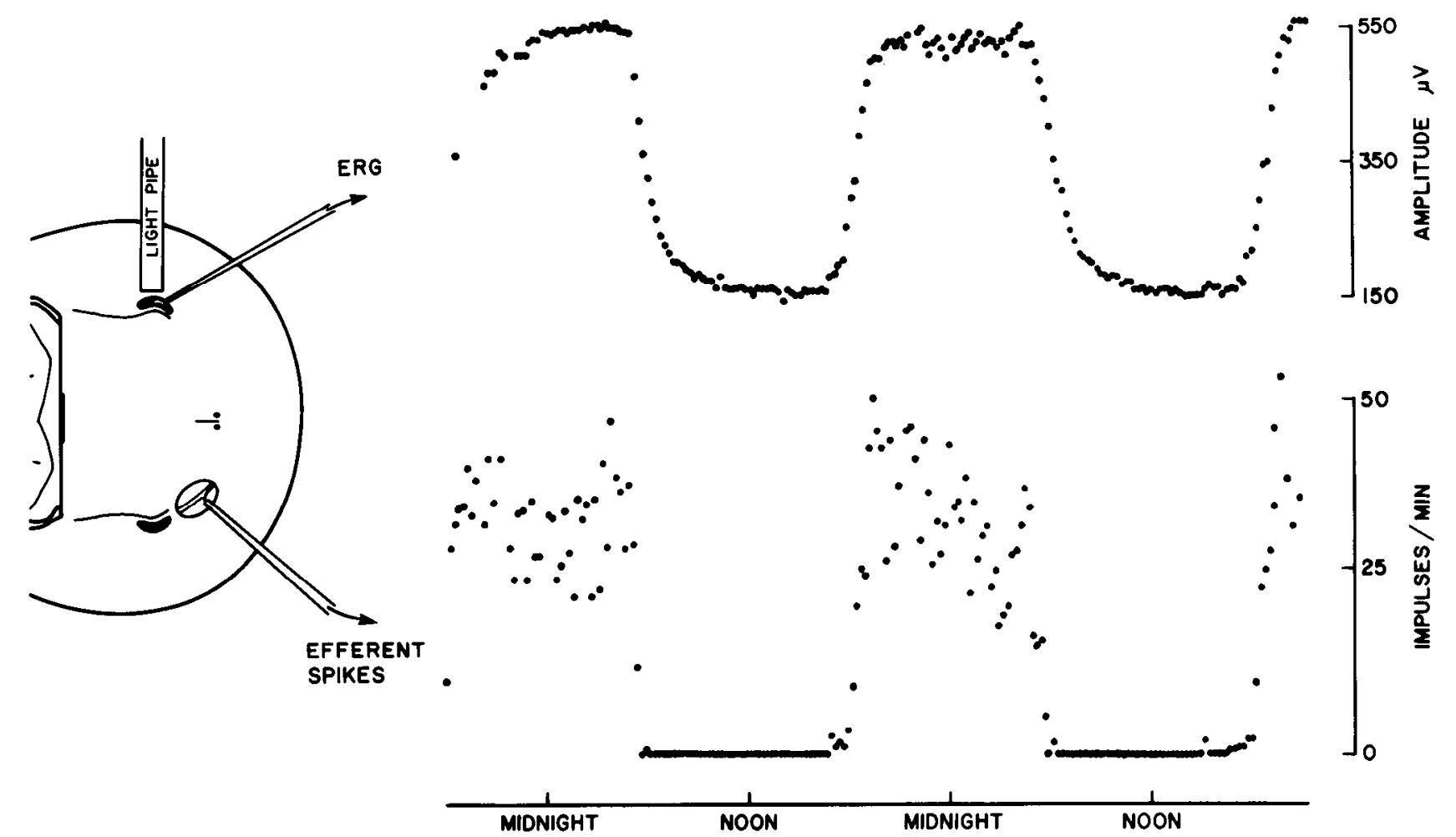

Figure 9. The circadian rhythm of efferent activity coincides with that of the ERG. On the left is a diagram showing the experimental arrangment for recording the ERG from the left lateral eye and efferent activity from the right lateral optic nerve. The ERGs were recorded and plotted as described earlier for Figures 1 and 6 . The efferent activity was recorded in situ as in the previous two figures, but the results are plotted as the number of impulses in a 2 -min period every 15 min. The appearance of efferent activity in the right optic nerve is strongly correlated with the increase in ERG amplitude of the left lateral eye. Both exhibit circadian rhythms of equal phase.

dim red light and placed in a recording chamber. Within $1 \mathrm{hr}$ of shock onset, the ERG amplitude increased to about $350 \mu \mathrm{V}$ as in $a$. However, the amplitude then began to decay and eventually reached the low, preshock level, while current pulses were still being delivered to the optic nerve trunk.

In sum, pulses of current delivered to the cut optic nerve mimic the effect of endogenous efferent activity on the ERG amplitude. Subsequent episodes of optic nerve shock may prove less effective, perhaps because efferent fibers in the cut optic nerve cease functioning or efferent terminals in the retina exhaust their supply of neurotransmitter. Optic nerve shock also becomes ineffective in the excised eye.

Circadian rhythm in lateral eye sensitivity. Figures 1 , 2,6 , and 9 show circadian changes in the response of the dark-adapted lateral eye. The corresponding changes in sensitivity can be determined from the function that relates response amplitude to light intensity. Figure 11 plots the amplitude of the ERG on the ordinate as a function of log light intensity on the abscissa. The day data were recorded from a lateral eye in situ at noon when the visual system was in the daytime state, and the night data were recorded $12 \mathrm{hr}$ later at midnight when the effects of the circadian efferent input to the eye were greatest. Both sets of measurements were carried out with the eye in the dark-adapted state.

The daytime data in Figure 11 (open circles) were fitted with a smooth curve by eye. The same curve shifted $1.3 \log$ units to the left and $0.1 \log$ units vertically, approximately overlaying the nighttime data (solid circles). A major portion of the increased sensitivity of the nighttime state to brief flashes of light causes a lateral shift of the intensity-response function. Such a shift is consistent with the idea that structural changes in the ommatidia at night allow photoreceptor cells to absorb more photons than during the day (Barlow et al., 1980a).

Both during the day and at night the ERG amplitude is graded with light intensity over a range of 5 to $6 \mathrm{log}$ units. At low levels of illumination the ERG amplitude is linearly related to test flash intensity over a range of about 2 log units. Because in this range a 10 -fold increase in intensity produces a 10-fold increase in amplitude of response, it is possible to use changes in ERG amplitude in this range as a measure of changes in sensitivity. For example, at $\log \mathrm{I}=-5$, the ERG amplitude increased from $3 \mu \mathrm{V}$ to $60 \mu \mathrm{V}$ at night. Because the response remained within the linear range, we can conclude that the sensitivity of the lateral eye increased 20 -fold from day to night. Experiments from other animals yielded 20 to 100 -fold increases in sensitivity.

At high levels of illumination the ERG response saturates and changes in sensitivity are not directly related to changes in response amplitude. The characteristics of response saturation are similar from animal to animal. At the highest test intensities $(\log I=0)$ the amplitude of the ERG response saturates at levels less than 1000 $\mu \mathrm{V}$.

Figure 12 shows that optic nerve stimulation can mimic the effect of the circadian clock on the intensity-response 
a
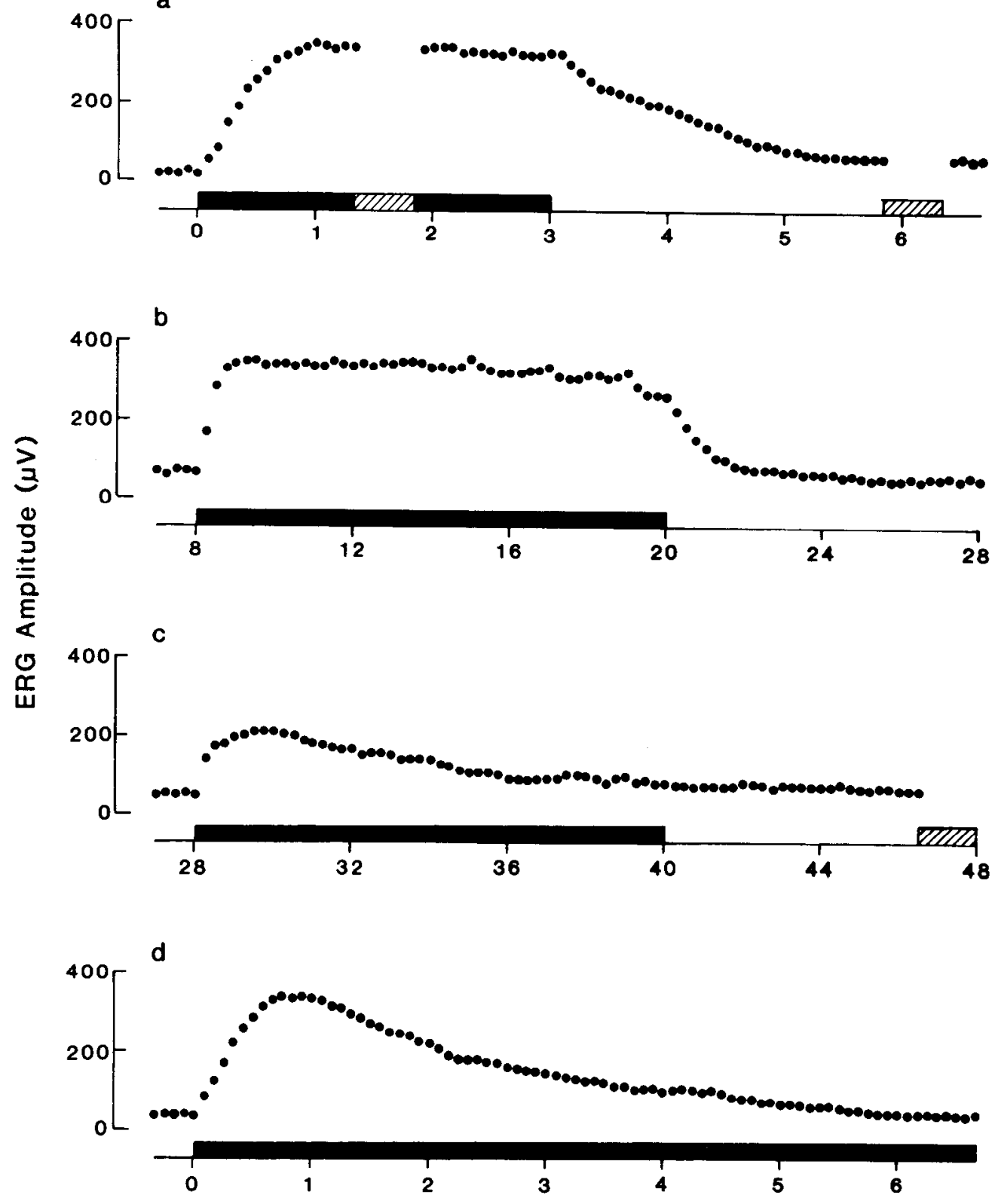

Hours

Figure 10. Optic nerve stimulation increases the ERG amplitude. $a$, Pulses of current delivered to the distal stump of the cut optic nerve in situ at a rate of $2 \mathrm{sec}^{-1}$ for $30 \mathrm{sec}$ every min increased the ERG 16 times the dark-adapted daytime level. After 3 hr the nerve shock was stopped, and the ERG amplitude decayed slowly to the daytime state. Cross-hatching indicates the times during which intensity-response functions were measured (see Fig. 12). $b$, A second episode of nerve shock in the same preparation. Note that the nerve shock was continued for $12 \mathrm{hr}$ and also that the time scale is compressed. $c$, A third episode of nerve shock for another $12 \mathrm{hr}$ in the same preparation. The ERG amplitude increased but not to the same levels as in $a$ and $b$. Also during the 12-hr period of shock the ERG amplitude decayed to the low daytime level. $a$, $b$, and $c$ were all carried out with the same preparation in the dark. $d$, Optic nerve shock can increase the ERG amplitude recorded from an excised lateral eye, but the efficacy of nerve shock declines rapidly. The eye was excised under dim red light and placed in a recording chamber. The current pulses and test flashes were the same as for the intact preparation in $a, b$, and $c$. The ERG initially increased to about $350 \mu \mathrm{V}$ but then declined to the low daytime levels. Repeated episodes of shock had no effect.

function of the lateral eye ERG. The data are taken from the experiment presented in Figure $10, a$ to $c$. The experimental conditions are identical to those in Figure 11, with the exception that the optic nerve was cut and the distal end of the cut nerve was drawn into a suction electrode. The open circles in Figure 12 give the inten- sity-response function of the eye in the dark-adapted state measured before stimulation of the optic neve, that is, before $0 \mathrm{hr}$ in Figure $10 \mathrm{a}$. Current pulses were then delivered to the cut nerve at the rate of $2 \mathrm{sec}^{-1}$ for $30 \mathrm{sec}$ every min. Eighty minutes later the ERG amplitude had reached maximal levels, and the experiment was inter- 


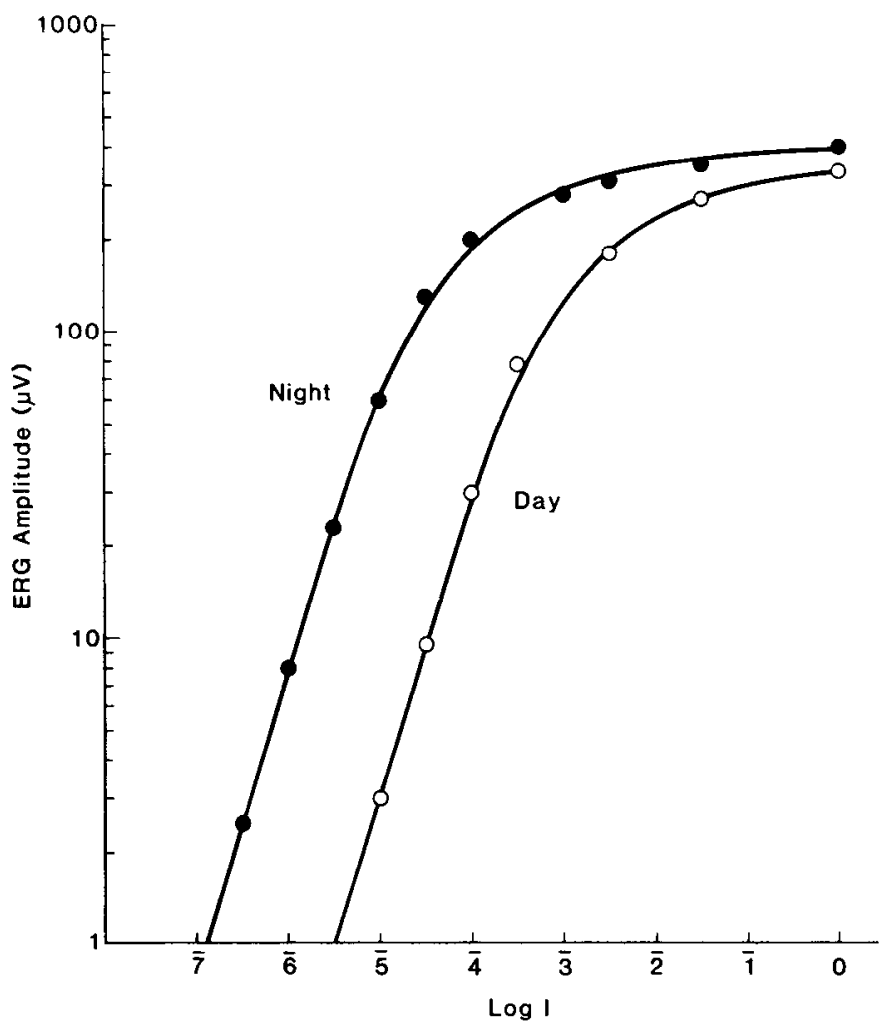

Figure 11. Intensity-response functions for the lateral eye ERG at night and during the day. Plotted on a log scale on the ordinate is the ERG amplitude as a function log light intensity plotted on the abscissa. Each response was evoked by a 100msec flash presented under dark-adapted conditions. The daytime data were recorded on the second day in darkness to avoid possible changes in the daytime levels (see Fig. 1). Both sets of data were fitted by the same curve moved $1.3 \mathrm{log}$ units on the abscissa and $0.1 \log$ units on the ordinate.

rupted (cross-hatching in Fig. 10a) for remeasurement of the intensity-response function (solid circles in Fig. 12). Nerve shock was turned off, and, after the ERG amplitude had decayed to a low, steady value (crosshatching at $6 \mathrm{hr}$ in Fig. 10a), the intensity-response function was again measured (triangles in Fig. 12).

Following the procedure in Figure 11, the data for the dark-adapted daytime state (labeled Before) were fitted by eye with a smooth curve. The same curve was shifted to the left and vertically to overlay the data measured During optic nerve shock. Finally, the curve was shifted back to the right and down to match the results recorded After shock offset. As was the case in Figure 11, the intensity-response functions in Figure 12 are linear over about a 2-log unit range at low light intensities and saturate at high intensities. The total range of graded response is about $6 \log$ units.

Optic nerve stimulation mimics in part the effects of endogenous, efferent aclivily by shifting the intensityresponse function without changing its shape. However, the shift in sensitivity caused by nerve shock generally exceeds that recorded from day to night. Nerve stimulation can increase lateral eye sensitivity by as much as 2 log units, whereas the average circadian changes in sensitivity are about $1.5 \mathrm{log}$ units. This is understandable because the shock regime used for the experiment in
Figure 12 delivers to the eye a higher level of efferent input than that normally exerted by the circadian clock. Note that the eye returned to a higher level of sensitivity after nerve shock. This is a common finding. Figure 1 shows that the dark-adapted ERG response during the first day is lower than during the second day. As indicated in Figure 12, the difference in sensitivity is about $0.5 \mathrm{log}$ unit. From this and other similar results I conclude that the effects of light adaptation cannot be completely reversed by an extended period of dark adaptation. Apparently efferent optic nerve activity is required to abolish the effects of light adaptation and achieve the highest level of retinal sensitivity at night.

\section{Discussion}

The circadian clock in the brain is a fundamental component of the Limulus visual system. It transmits efferent optic nerve activity at night to the lateral eyes, median ocelli, and ventral photoreceptors (Eisele et al., 1982). The efferent input to the lateral eyes exerts multiple physiological and anatomical effects that combine

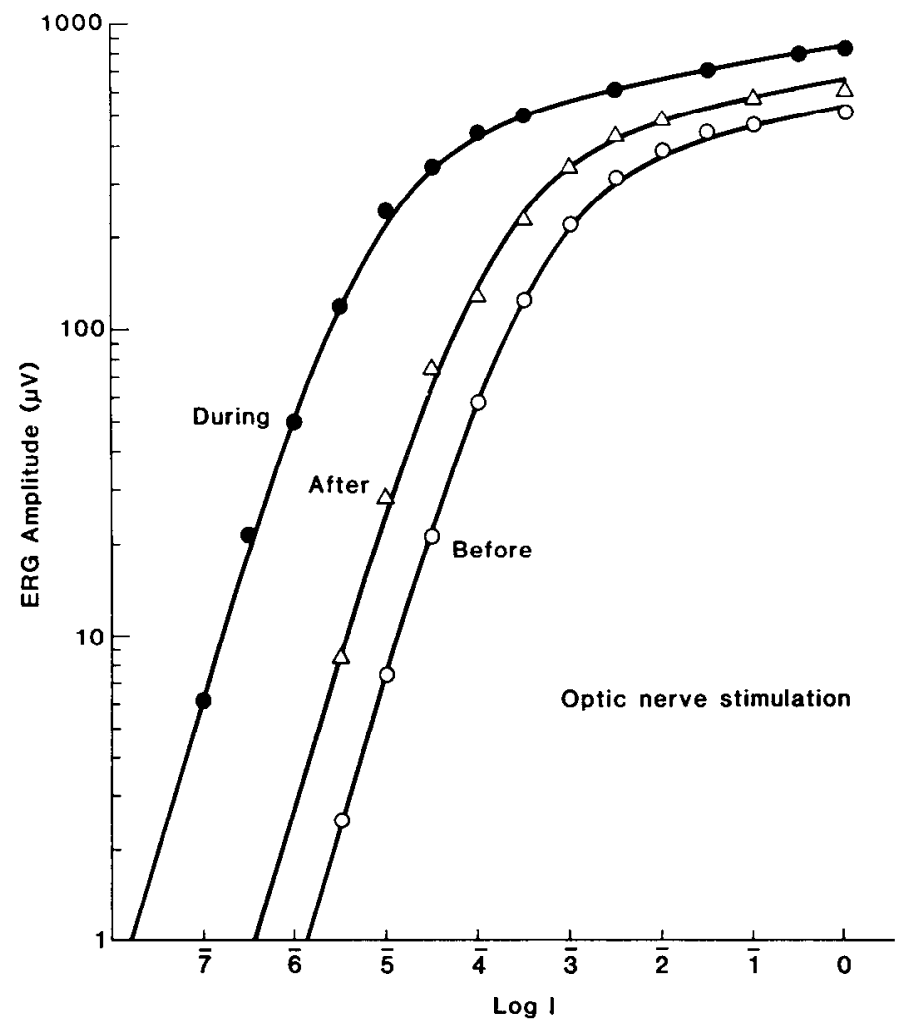

Figure 12. Optic nerve stimulation reproduces the effect of the circadian clock on lateral eye sensitivity. The coordinates and experimental conditions were the same as in Figure 11, with the exception that the optic nerve was cut and the distal end was pulled into a suction electrode. The data are taken from the experiment presented in Figure $10 a$ to $c$. All measurements were carried out during the day. After the preparation was dark adapted and before $0 \mathrm{hr}$ in Fig. 10a, an intensityresponse function was measured (labeled Before). Current pulses were turned on and $1.3 \mathrm{hr}$ later, after the ERG amplitude reached maximal levels, the intensity-response function was measured again (labeled During). Nerve shock was turned off and $3 \mathrm{hr}$ later, after the ERG amplitude reached a low steady level, the function was remeasured (labeled After). Each set of data was fitted by the same curve shifted parallel to the axes. 
to increase retinal sensitivity. The efferent input to the median ocelli increase their sensitivity in the visible range $(400$ to $700 \mathrm{~nm}$ ) but not in the ultraviolet (330 to $400 \mathrm{~nm}$ ) to which they are most sensitive (Barlow et al., $1980 \mathrm{~b})$. The efferent input to the ventral photoreceptors has no known effect (Kaplan et al., 1980). The circadian clock also mediates interactions among the photoreceptor organs, but only at night (Barlow et al., 1980b). Although our knowledge of the entire visual system is far from complete (Chamberlain and Barlow, 1980, 1982), it is clear that the animal has evolved numerous mechanisms for adapting visual sensitivity to periodic changes in ambient illumination. A key to understanding the mechanism and the underlying organization of the visual system is the circadian clock.

Properties of the circadian clock. Circadian clocks generate rhythms that generally persist under constant environmental conditions. When Limulus is maintained in darkness, the circadian rhythm in ERG amplitude continues without attenuation for at least 1 year. In Figure $2 b$ the pattern of changes in ERG amplitude recorded after 1 year is similar to that recorded at the beginning and middle of the year (data not shown). Also, the circadian period of $23.4 \mathrm{hr}$ did not change significantly over the course of the year.

Figure 2 gives examples of circadian rhythms from two animals having periods longer and shorter than $24 \mathrm{hr}$. Measurements from 75 Limuli yield periods ranging from 22.2 to $25.5 \mathrm{hr}$, with a mean value of $23.9 \pm 0.7 \mathrm{hr}$. The period length does not differ significantly from $24 \mathrm{hr}$ and does not seem to be correlated with any exogenous factors, such as temperaturc, time of day, tidal variations, etc. Studies of circadian phenomena of other animals have uncovered a general relationship between the behavior of an animal and the circadian period (Aschoff, 1960). Aschoff's rule states that nocturnally active animals exhibit circadian rhythms with periods $<24 \mathrm{hr}$ when maintained in constant darkness and periods $>24 \mathrm{hr}$ when exposed to constant light. Although studies of Limulus activity cycles are limited, they indicate that the animals are predominantly active at night (Barlow and Palfai, 1971). Thus Limulus may be expected to exhibit a circadian period $<24 \mathrm{hr}$ for conditions of constant darkness. 'The $\mathrm{ERG}$ data reported here yield a mean value of about $24 \mathrm{hr}$ for constant darkness, but no measurements have yet been made for conditions of constant illumination. It remains to be seen whether Limulus adheres to Aschoff's rule.

Circadian clocks entrain to the daily cycle of sunlight. They entrain by adjusting their phase in response to the external light cycle. Phase-shifting sensitivity can be analyzed by measuring the magnitude and sign of phase shifts induced by light flashes presented at various times during a circadian cycle that is free running in constant darkness. Figure 5 shows that flashes presented to $\mathrm{Lim}$ ulus between subjective dusk and midnight (circadian time (CT) 12 to $18 \mathrm{hr}$ ) elicited maximum phase delays, and those presented between midnight and dawn (CT 18 to $24 \mathrm{hr}$ ) elicited maximum phase advances. Flashes at midnight (CT $18 \mathrm{hr}$ ) did not shift the phase. Such phase shifts are characteristic of a number of circadian systems (Pittendrigh, 1965, 1981).
Both the phase advances and phase delays in Figure 5 occurred rapidly within $24 \mathrm{hr}$, which is uncharacteristic of phase shifts of circadian rhythms in multicellular organisms (Pittendrigh and Bruce, 1959). Phase delays are generally rapid, whereas phase advances often require several days to complete. Such transient behavior was not observed for Limulus.

Which visual organs entrain the circadian clock? All of the phase shifts in Figure 5 occurred in response to illumination of the entire animal or of the lateral eyes alone. Restricting illumination to the median ocelli was not effective. A third photosensitive organ, the ventral photoreceptors, was not tested. The lateral compound eyes, which are the principal eyes for mediating pattern vision, may also be the primary organs for entraining the clock.

Neural connections between the clock and the visual system. All major visual organs-lateral eyes, median ocelli, and ventral photoreceptors-receive efferent nerve signals from the clock at night (Eisele et al., 1982). The efferent signals are transmitted to the lateral eyes and ventral photoreceptors by small fibers in the optic nerve trunks (Clark et al., 1969; Fahrenbach, 1971, 1973). In the lateral eye the efferent fibers branch profusely and terminate on retinular, eccentric, and pigment cell bodies (Fahrenbach, 1981), as well as on collaterals of eccentric cells in the lateral plexus (W. H. Fahrenbach, personal communication). In the ventral photoreceptor organ the efferent fibers branch and terminate on photoreceptor cells (Clark et al., 1969; Calman and Chamberlain, 1982). No information is yet available on the anatomy of the efferent pathway to the median ocelli.

Efferent cell bodies and the neural circuitry of the clock are located in the protocerebrum of the brain (Eisele et al., 1982). This result is based on long term recordings of efferent optic nerve activity from isolated segments of the excised brain in organ culture. Although the precise location of the clock and associated efferent cell bodics has not yet been determined, anatomical and physiological data point to the medulla of the protocerebrum (Chamberlain and Barlow, 1980; Eisele et al., 1982).

It seems unlikely that the efferent cell bodies themselves contain the circadian pacemaker. Preliminary physiological data suggest that the circadian clock and efferent cell bodies form a neural circuit. The complexity is exemplified by the finding that illumination of the median ocelli can modulate lateral eye sensitivity by enhancing the efferent output of the clock, but only at night (Barlow et al., 1980b). During the day, illumination of the ocelli is generally not effective. Apparently the clock "gates" the ocellar influence on lateral eye sensitivity. No reciprocal effects have been observed, that is, lateral eye illumination does not modulate median ocellar sensitivity, day or night. On the other hand, the lateral eyes can entrain the clock and can also modulate the efferent input to one another (R. Barlow, unpublished results). Neural interactions between the lateral eyes are complex and not well understood. They are also gated by the clock: they occur only at night. Further evidence for the complexity of the neural circuit comes from the nature of the efferent activity. 
Efferent optic nerve activity. The clock generates efferent optic nerve activity at night (Figs. 8 and 9). The activity is characterized by repetitive bursts of impulses fired by as many as 10 to 20 efferent fibers in each nerve trunk. It is interesting to note that bursting pacemaker activity is characteristic of an identified neurosecretory cell in mollusc (Gainer, 1972) and that the efferent neurons in Limulus seem to be neurosecretory (Fahrenbach, 1971, 1981).

The repetitive bursts of impulses indicate that the efferent cell bodies in the brain are either coupled together or receive nearly synchronous inputs from the circadian clock. The bursts become less distinct in the early morning hours, suggesting that the synchrony changes during the circadian cycle. The efferent activity in Figurc 8 was recorded from a single optic nerve trunk. Simultaneous recordings from both nerve trunks (Fig. 7) show synchronous activity among the efferent fibers from opposite sides of the brain. The nature of efferent activity recorded at the periphery points to a high degree of coupling among the central neurons that comprise the clock circuitry.

For example, the synchrony between the efferent activity of the right and left optic nerves may result from the cells of origin in the brain being driven by a single clock or by separate clocks coupled together. Recordings from the excised brain in organ culture (Eisele et al., 1982) suggest the existence of separate clocks. In these experiments we found that partially bissecting the protocerebrum of the brain abolished the synchrony but not the overall activity. Thus the brain may contain two cirdadian clocks located in opposite halves of the protocerebrum and coupled via neural connectives. Although knowledge of the wiring of the brain is sparse, it is clear that the circadian clock and its associated neural circuitry are key elements in the organization of the visual system.

Efferent neurotransmitter. In view of the profound influence of the circadian clock on retinal function, several studies have focused on identifying the neurotransmitter. Initial pharmacological experiments point to serotonin (Barlow et al., 1977); however, the dose levels were relatively high and subsequent experiments could not detect either endogenous serotonin or the synthesis of serotonin in the retina. Battelle (1980) detected octopamine in several loci of the Limulus visual system and suggested the possibility that the amine may be involved in the regulation of circadian rhythms. Autoradiographic analyses have localized octopamine in the efferent terminals of the lateral eye (Battelle et al., 1982). Pharmacological studies show that octopamine can mimic in part the anatomical and physiological changes induced in the retina by the clock and that an octopamine antagonist can reversibly reduce the action of the clock (Kass and Barlow, 1981, 1982). In sum, all evidence points to octopamine as an efferent neurotransmitter in the retina.

Clock's influence on the visual system. As reported here, the clock generates circadian rhythms in the amplitude of the ERG of both the lateral eyes and median ocelli (Fig. 6). Reported elsewhere are the clock's influence on the quantum catch and noise of single photoreceptors (Barlow et al., 1977; Kaplan and Barlow, 1980), on the anatomy of retinal cells (Barlow et al., 1980a), on the daily renewal of photoreceptor membrane, and on some properties of lateral inhibition (Batra and Barlow, 1982). The clock's influence on the visual system exhibits seasonal changes that adjust the efferent output to fluctuations in day length (Barlow, 1982). These studies focused on the lateral eyes. Such detailed analyses have not yet been carried out for the median ocelli. As indicated earlier, the ventral photoreceptors also receive an efferent input, but initial studies have not detected circadian phenomena (Kaplan et al., 1980).

The circadian change in ERG amplitude of the lateral eye produces a nearly parallel shift of about $1.5 \mathrm{log}$ units in the intensity-response function (Fig. 11). Such a shift can be accounted for by an increase in the total number of photons caught by the photoreceptors. This interpretation is consistent with the known circadian changes in the morphology of the rhabdom and in the aperture between the corneal lens and the photoreceptors (Barlow et al., 1980a). Although not reported here, similar nighttime shifts occur in the intensity-response function of the median ocellar ERG. The interpretation of circadian changes in ocellar responses must await future studies.

Clock's influence on visually guided behavior. What role do circadian changes in visual sensitivity play in Limulus behavior? The only well documented behavior exhibited by the animal in its natural habitat is mating. In the spring along the eastern coast of North America, Limuli move in from deep water and build nests on protected beaches near the water's edge at high tide (Lockwood, 1870). The activity occurs both day and night, and recent experiments with painted cement castings of the female shell indicate that vision plays a role (Barlow et al., 1982). High visual sensitivity generated by the clock at night may adapt the animal for nocturnal mating behavior. It should also be noted that behavioral measures of circadian changes in visual sensitivity carried oul in the laboratory correspond well with the physiological changes presented in this paper (Powers and Barlow, 1981).

Circadian rhythms in other visual systems. Circadian rhythms seem to be characteristic of invertebrate visual systems (Welsh, 1938; Jahn and Wulff, 1943; Aréchiga and Wiersma, 1969; Page, 1981). Circadian migration of retinal screening pigments is generally believed to underlie the endogenous rhythms in visual response (Jahn and Crescitelli, 1940; Fleissner, 1972). Of the numerous animals that have been studied, scorpion and orb spider seem to share the most features with Limulus. Pigment movements within photoreceptor cells of the median ocelli of scorpion exhibit a circadian rhythm (Fleissner, 1972) which is blocked by sectioning the optic nerve (Fleissner and Fleissner, 1978). Efferent cell bodies are located in both halves of the scorpion brain (Fleissner and Heinrichs, 1982), suggesting the possibility of bilateral circadian oscillators. The anterior median ocelli of orb spiders exhibit circadian changes in sensitivity (Yamashita and Tateda, 1978) which seem to be controlled by efferent optic nerve activity (Yamashita and Tateda, 1981).

In some animals the control of circadian rhythms may be more complex. For example, both neural and humoral processes seem to mediate the circadian rhythms in ERG 
amplitude in crayfish (Aréchiga and Wiersma, 1969). The amplitude of the retinal responses is modulated by a hormone released from the sinus gland in the eyestalk (Aréchiga el al., 1974). Apparently hormone release is triggered by the activity of central visual pathways because sectioning the optic nerve abolishes the endogenous ERG rhythm (Page and Larimer, 1975). In Aplysia the eye itself contains a circadian clock that generates rhythms of spontaneous optic nerve activity (Jacklett, 1969). The eye receives an efferent input from the brain (Eskin, 1977) which may be involved in resetting the clock in the eye (Prichard and Lickey, 1981). Circadian rhythms in response have been recorded from single interneurons located in central visual pathways of the bee (Kaiser, 1979). During the day, lobular neurons in the bee brain respond strongly to pattern movement in the visual field; at night, the response is weak. It is apparent that endogenous circadian clocks modulate visual processing in a broad range of invertebrate species.

Circadian rhythms have also been detected in several vertebrate visual systems. A striking example is the pigeon which exhibits a nighttime increase in the amplitude of the ERG response to short wavelength stimuli when the animal is maintained in darkness (Barattini et al., 1981). The results suggest the possibility that a circadian clock shifts the functional dominance of rods and cones in the retina to adapt the system to periodic fluctuations in the photic environment. Another example is the diurnal lizard which exhibits a daytime increase in the ERG amplitude when maintained under constant conditions (Fowkles et al., 1982). The teleost retina exhibits striking circadian changes in retinomotor activity (John et al., 1967; Ali, 1975; Levinson and Burnside, 1981). Comparable changes in visual sensitivity have been detected in recent behavioral studies of goldfish (M. K. Powers, personal communication).

Studies of membrane turnover in mammalian photoreceptors show that the renewal process in the rat retina exhibits a circadian rhythm when the animal is maintained in constant darkness (La Vail, 1976). The underlying circadian oscillator seems to be located in the retina with the phase resetting mechanism in the brain (Tierstein et al., 1980). Finally, preliminary studies suggest the possible existence of cyclic changes in human visual sensitivity (Powers et al., 1982).

\section{References}

Ali, M. A. (1975) Retinomotor responses. In Vision in Fishes. New Approaches in Research, M. A. Ali, ed., pp. 313-355, Plenum Press, New York.

Aréchiga, H., and C. A. G. Wiersma (1969) Circadian rhythm of responsiveness in crayfish visual units. J. Neurobiol. 1: 7185.

Aréchiga, H., A. Hubernan, and E. Naylor (1974) Hormonal modulation of circadian neural activity in Carcinus maenus (L). Proc. R. Soc. Lond. B 187: 299-313.

Aschoff, J. (1960) Exogenous and endogenous components in circadian rhythms. Cold Spring Harbor Symp. Quant. Biol. 25: 11-28.

Barattini, S., B. Battisti, L. Cervetto, and P. Marroni (1981) Diurnal changes in the pigeon electroretinogram. Rev. Can. Biol. 40: 133-137.

Barlow, R. B., Jr. (1982) Seasonal changes in the circadian modulation of sensitivity of the Limulus lateral eye. Biol Bull. 163: 380.

Barlow R. B., Jr., and S. C. Chamberlain (1980) Light and a circadian clock modulate structure and function in Limulus photoreceptors. In The Effects of Constant Light on Visual Processes, T. P. Williams and B. N. Baker, eds., pp. 247-269, Plenum Press, New York.

Barlow, R. B., Jr., and A. J. Fraioli (1978) Inhibition in the Limulus lateral eye. J. Gen. Physiol. 71: 699-720.

Barlow, R. B., Jr., and T. Palfai (1971) Conditioning of Limulus behavior. In Semiannual Research Report LSC.15, p. 3 , Laboratory of Sensory Communication, Syracuse University, Syracuse, NY.

Barlow, R. B., Jr., S. J. Bolanowski, Jr., and M. L. Brachman (1977) Efferent optic nerve fibers mediate circadian rhythms in the Limulus eye. Science 197: 86-89.

Barlow, R. B., Jr., S. C. Chamberlain, and J. Z. Levinson (1980a) Limulus brain modulates the structure and function of the lateral eves. Science 210: 1037-1039

Barlow, R. B., Jr., S. C. Chamberlain, S. J. Bolanowski, Jr., L. A. Galway, Jr., and D. P. Joseph (1980b) One eye can increase the sensitivity of another in Limulus. Soc. Neurosci. Abstr. 6: 344 .

Barlow, R. B., Jr., L. C. Ireland, and I. Kass (1982) Inhibition in the Limulus lateral eye. Nature Lond. 296: 65-66.

Batra, R., and R. B. Barlow, Jr. (1982) Efferent control of pattern vision in Limulus. Soc. Neurosci. Abstr. 8: 49.

Battelle, B. -A. (1980) Neurotransmitter candidates in the visual system of Limulus polyphemus: Synthesis and distribution of octopamine. Vision Res. 20: 911-922.

Batelle, B. -A., J. A. Evans, and S. C. Chamberlain (1982) Efferent fibers to Limulus eyes synthesize and release octopamine. Science 216: 1250-1252.

Bayer, D. S., and R. B. Barlow, Jr. (1978) Limulus ventral eye: Physiological properties of photoreceptor cells in an organ culture medium. J. Gen. Physiol. 72: 539-563.

Calman, B. G., and S. C. Chamberlain (1982) Distinct lobes of Limulus ventral photoreceptors. II. Structure and ultrastructure. J. Gen. Physiol. 80: 839-862.

Chamberlain, S. C., and Barlow, R. B., Jr. (1977) Morphological correlates of efferent circadian activity and light adaptation in the Limulus lateral eye. Biol. Bull. 153: 418-419.

Chamberlain, S. C., and R. B. Barlow, Jr. (1979) Light and efferent activity control rhabdom turnover in Limulus photoreceptors. Science 206: 361-363.

Chamberlain, S. C., and Barlow, R. B., Jr. (1980) Neuroanatomy of the visual afferents in the horseshoe crab (Limulus poly. phemus). J. Comp. Neurol. 192: 387-400.

Chamberlain, S. C., and R. B. Barlow, Jr. (1982) Retinotopic organization of lateral eye input to Limulus brain. J. Neurophysiol. 48: 505-520.

Clark, A. W., R. Millecchia, and A. Mauro (1969) The ventral photoreceptor cells of Limulus. I. The microanatomy. J. Gen. Physiol. 54: 289-309.

Eisele, L. E., L. Kass, and R. B. Barlow, Jr. (1982) Circadian clock generates optic nerve activity in the excised Limulus brain. Biol. Bull. 163: 382.

Eskin, A. (1977) Neurophysiological mechanisms involved in photo-entrainment of the circadian rhythm from the Aplysia eye. J. Neurobiol. 8: 273-299.

Fahrenbach, W. H. (1971) The morphology of the Limulus visual system. IV. The lateral optic nerve. Z. Zellforsch. 114: 532-545.

Fahrenbach, W. H. (1973) The morphology of the Limulus visual system. V. Protocerebral neurosecretion and ocular innervation. Z. Zellforsch. 144: 153-166.

Fahrenbach, W. H. (1981) The morphology of the Limulus visual system. VII. Innervation of photoreceptor neurons by 
neurosecretory efferents. Cell Tissue Res. 216: 655-659.

Fleissner, G. (1972) Circadian sensitivity changes in the median eyes of the North African scorpion, Androctonus australis. In Information Processing in the Visual System of Arthropods, R. Wehner, ed., pp. 133-139, Springer, New York.

Fleissner, G., and G. Fleissner (1978) The optic nerve mediates the circadian pigment migration in the median eyes of the scorpion. Comp. Biochem. Physiol. (A) 61: 69-71.

Fleissner, G., and S. Heinrichs (1982) Neurosecretory cells in the circadian-clock system of the scorpion, Androctonus australis. Cell Tissue Res. 224: 233-238.

Fowkles, D. H., C. J. Karwoski, and L. M. Proenza (1982) ERG circadian rhythm in the free-moving lizard (anolis Carolinensis). Invest. Ophthalmol. Vis. Sci. 22 (Suppl.): 281.

Gainer, H. (1972) Electrophysiological behavior of an endogenously active neurosecretory cell. Brain Res. 39: 403-418.

Hartline, H. K. (1935) The discharge of nerve impulses from the single visual sense cell. Cold Spring Harbor Symp. Quant. Biol. 3: 245-250.

Jacklett, J. W. (1969) Circadian rhythm of optic nerve impulses recorded in darkness from isolated eye of Aplysia. Science 164: $562-563$.

Jahn, T. L., and F. Crescitelli (1940) Diurnal changes in the electrical response of the compound eye. Biol. Bull. 78: 4252.

Jahn, T. L., and V. J. Wulff (1943) Electrical aspects of a diurnal rhythm in the eye of Dytiscus fasciventris. Physiol. Zool. 16: 101-9.

John, K. R., M. Segal, and L. Zawatsky (1967) Retinomotor rhythms in the goldfish, Carassius auratus. Biol. Bull. 15:2: 200-210.

Kaiser, W. (1979) Circadian variations in the sensitivity of single visual interneurones of the bee, Apis mellifica carnica. Verh. Dtsch. Zool. Ges. 72: 211.

Kaplan, E., and R. B. Barlow, Jr. (1975) Properties of visual cells in the lateral eye of Limulus in situ. Extracellular recordings. J. Gen. Physiol. 66: 303-326.

Kaplan, E., and R. B. Barlow, Jr. (1980) Circadian clock in Limulus brain increases response and decreases noise of retinal photoreceptors. Nature 286: 393-395.

Kaplan, E., R. Batra, and R. B. Barlow, Jr. (1980) Recording from the Limulus ventral eye in situ: Is there a circadian rhythm? Biol. Bull. 159: 486.

Kass, L., and R. B. Barlow, Jr. (1981) Octopamine increases the ERG of the Limulus lateral eye. Biol. Bull. 159: 487.

Kass, L., and R. B. Barlow, Jr. (1982) Efferent neurotransmission of circadian rhythms in Limulus lateral eye: Single cell studies. Biol. Bull. 163: 386.
La Vail, M. M. (1976) Rod outer segment disk shedding in rat retina: Relationship to cyclic lighting. Science 194: 10711074.

Levinson, G., and B. Burnside (1981) Circadian rhythms in teleost retinomotor movements. Invest. Ophthalmol. Vis. Sci. 20: 294-303.

Lockwood, S. (1870) The horse foot crab. Am. Nat. 4: 257-274.

Page, T. L. (1981) Neural and endocrine control of circadian rhythmicity in invertebrates. In Handbook of Behavioral Neurobiology Vol. 4, Biological Rhythms, J. Aschoff, ed., pp. 145-172, Plenum Press, New York.

Page, T. L., and J. L. Larimer (1975) Neural control of circadian rhythmicity in the crayfish. II. The ERG amplitude rhythm. J. Comp. Physiol. 97: 81-96.

Pittendrigh, C. S. (1965) On the mechanism of the entrainment of a circadian rhythm by light cycles. In Circadian Clocks, J. Aschoff, ed., pp. 277-297, North-Holland, Amsterdam.

Pittendrigh, C. S. (1981) Circadian systems: Entrainment. In Handbook of Behavioral Neurobiology, Vol. 4, Biological Rhythms, J. Aschoff, ed., pp. 95-124, Plenum Press, New York.

Pittendrigh, C. S. and V. G. Bruce (1969) Daily rhythms as coupled oscillator systems and their relation to thermoperiodism and photoperiodism. In Photoperiodism and Related Phenomena in Plants and Animals, R. B. Withrow, ed., pp. 475-505, American Association for the Advancement of Science, Washington, D.C.

Powers, M. K., and R. B. Barlow, Jr. (1981) Circadian changes in visual sensitivity of Limulus: Behavioral evidence. Biol. Bull. 161: 350(A).

Powers, M. K., C. J. Bassi, and C. L. Rosen (1982) Daily fluctuations in absolute visual sensitivity. Invest. Ophthalmol. Vis. Sci. 22: (Suppl.): 127.

Prichard, R. G., and M. E. Lickey (1981) In vitro resetting of the circadian clock in the Aplysia eye. J. Neurosci. 1: 835839.

Teirstein, P. S., A. I. Goldman, and P. J. O'Brien (1980) Evidence for both local and central regulation of rat rod outer segment disc shedding. Invest. Ophthalmol. Vis Sci. 19: 12681273.

Welsh, J. H. (1938) Diurnal rhythms. Q. Rev. Biol. 13: 123-139.

Yamashita, S., and H. Tateda (1978) Spectral sensitivities of the anterior median eyes of the orb web spiders, Argiope bruennichii and $A$. amoena. J. Exp. Biol. 74: 47-57.

Yamashita, S., and H. Tateda (1981) Efferent neural control in the eyes of orb weaving spiders. J. Comp. Physiol. (A) 143: $477-483$ 